\title{
Optical Analyses of Si and GaAs Semiconductors by Fractional-Derivative-Spectrum Methods
}

\author{
W. Rzodkiewicz ${ }^{a *}$, M. Kulik ${ }^{b}$, E. PAPIS $^{a}$ And A. Szerling ${ }^{a}$ \\ ${ }^{a}$ Institute of Electron Technology, al. Lotników 32/46, 02-668 Warsaw, Poland \\ ${ }^{b}$ Institute of Physics, UMCS, pl. M.Curie-Skłodowskiej 1, 20-031 Lublin, Poland
}

\begin{abstract}
Optical spectra analysis provides a wealth of information on physical properties of various semiconductor materials. Fractional derivative spectrum technique is especially interesting when the limitations of the standard treatment occur. In this paper we present the fractional derivative spectrum method for analysis of the optical spectra for both Si and GaAs. The significant changes in critical point parameters in each treated Si and GaAs samples in comparison to that before treatment have been observed. Our investigation illustrates that fractional derivative spectrum is a very good technique to extract basic information on relevant physical quantities from the observed optical spectra, and it has the advantages of flexibility, directness, and sensitivity, which give possibility to obtain the Van Hove singularities (critical point parameters) efficiently with one consent.
\end{abstract}

PACS numbers: 78.40.Fy, 64.60.-fd, 71.20.-b

\section{Introduction}

The analysis of optical spectra provides a wealth of information about electronic structures of solids. Dielectric function $\varepsilon$ is widely used for this purpose, since it gives the optical responses of solids under an external field.

Spectroscopic ellipsometry (SE) is a terrific technique to investigate the optical response of solids [1]. Particularly, the above mentioned method allows the accurate determination of the spectral dependence of $\varepsilon$ on the photon energy $E$. On the basis of these characteristics in the combination with fractional-derivative-spectrum (FDS) model [2], the critical points (CP) can be defined with one consent. This eases the extraction of the parameters that depict the interband electronic transitions by means of a line shape analysis.

FDS technique is especially interesting when the limitations of the standard treatment occur. Thus, in this paper we describe FDS model for analysis of the optical spectra for both Si and GaAs semiconductor materials before and after treatment.

\section{Fractional-derivative-spectrum method}

First time, He and Mo applied the fractional-dimensional space model to the optical transitions in the vicinity of critical points (called as van Hove singularities). We can observe these singularities in the dielectric function spectrum. So far, the following line shape of an integer dimensional model has been used for analyses of the interband transitions [3-5]:

$$
\varepsilon(E)=C-A \mathrm{e}^{\mathrm{i} \phi}\left(E-E_{\mathrm{g}}+\mathrm{i} \Gamma\right)^{n / 2-1},
$$

where the $\mathrm{CP}$ is described by amplitude $A$, the exci-

\footnotetext{
* corresponding author; e-mail: rzodki@ite.waw.pl
}

tonic phase angle $\phi$, the energy gap at critical point (the threshold energy) $E_{\mathrm{g}}$, Lorentzian broadening parameter $\Gamma$. The parameter $n$ (integer between 0 and 3) stands for the dimensionality of the CP. The above-mentioned formula gives the well-known standard treatment for studying a critical point transition. The above technique has been widely applied for the critical parameters from optical spectra, though it has some restrictions. First of all, to extract all the CP parameters, a model line shape must be assumed in respect of a selected segment of the spectrum which allows for the structure of interest. Except this, the second or third derivative of a spectrum is mainly engaged to enhance a $\mathrm{CP}$ structure [6]. Obviously, it is connected with the fact that differentiation also heightens noise, and the special smoothing technique is needed. Hence, it can cause distortion of the informational content of the data. In the standard treatment, it is hard to decide which model is the best. Because, when the real dimensionality of a CP is fractional (e.g. 1.5), both $1 \mathrm{D}$ and $2 \mathrm{D}$ models may portray comparable fitting results by least-squares regression. In the literature [3], it was shown that the dielectric function for direct interband transitions of $n$-dimensional critical point electrons can be precisely given as the $n / 2$-th iterated integrals of the dielectric function of the harmonic oscillator.

Generally speaking, the complex dielectric function with Lorentzian broadening for direct allowed interband transitions can be given by

$$
\varepsilon(E)=\left(M^{2} / E^{2}\right)(1 / \pi) \int G(E) \mathrm{d} E /\left(E_{\mathrm{g}}-E-\mathrm{i} \Gamma\right),
$$

where $M$ stands for the momentum matrix element, $G(E) \neq 0$ is the joint density of (JDOS) states as a function of energy. The factor $M$ can be obtained from the vector product of amplitude $A$ and Lorentzian broadening parameter $\Gamma(2)$. For various types of critical points, definition of the dielectric function given by $\mathrm{He}$ and Mo 
in [3] can be presented as follows:

$$
\begin{aligned}
& \varepsilon(E)=\left(M^{2} / E^{2}\right) \mathrm{i}^{r-\alpha} \Gamma(\alpha / 2)\left(d^{-\alpha / 2} / \mathrm{d} E^{-\alpha / 2}\right) \\
& \quad /\left(E_{\mathrm{g}}-E-\mathrm{i} \Gamma\right)
\end{aligned}
$$

where $r$ denotes the type (order) of critical point, $\alpha$ is the real dimensionality (usually fractional) of the $\mathrm{CP}$, and $\Gamma(\alpha / 2)$ stands for the gamma function as well as the last factor $\left(\mathrm{d}^{-\alpha / 2} / \mathrm{d} E^{-\alpha / 2}\right)\left[1 /\left(E_{\mathrm{g}}-E-\mathrm{i} \Gamma\right)\right]$ is the conventional symbol expressing the $\alpha / 2$-th iterated integrals. It is worth emphasizing that the above definition is equivalent to formula (1) when $\alpha$ is the integer $n$. The algorithm which was used in our calculation of fractional differentiation of the investigated spectra is presented below [7]:

$$
\begin{aligned}
& \frac{\mathrm{d}^{q} f}{\mathrm{~d}^{q} x}=\lim _{\mathrm{N} \rightarrow \infty}\left\{\frac{x^{-q}}{\Gamma(-q)} N^{q} \sum_{j=0}^{\mathrm{N}-1} \frac{\Gamma(j-q)}{\Gamma(j+1)}\right. \\
& \left.\quad \times f\left(x+\frac{q x}{2 N}-\frac{j x}{N}\right)\right\},
\end{aligned}
$$

where $q$ is the real value (in the case of differentiation of spectra, positive value is used), $f$ denotes the function representing the investigated spectra $\left(f_{1}=E^{2} \varepsilon_{1}\right.$ or $\left.f_{2}=E^{2} \varepsilon_{2}\right), x$ is the corresponding value of energy, and $N$ stands for the number of elements which were used in the investigated energy range. For the sake of fractional order, the algorithm presented here is the most accurate algorithm [7] used for calculations of derivatives and integrals so far.

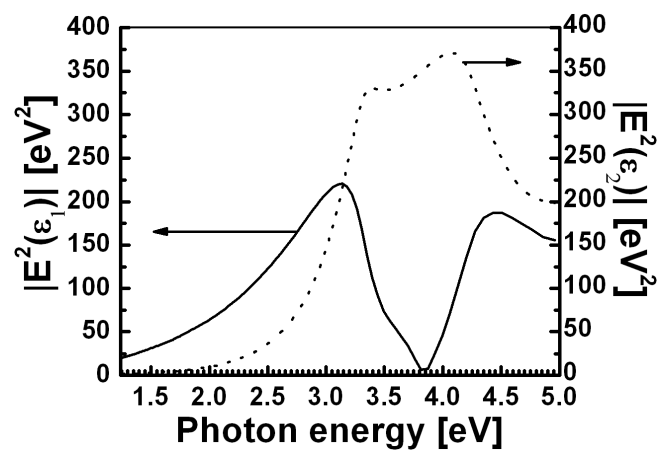

Fig. 1. Spectra $\left|\varepsilon_{1} E^{2}\right|$ and $\left|\varepsilon_{2} E^{2}\right|$ as a function of photon energy for nitrogen implanted silicon substrates.

The analyzed spectra $E^{2} \varepsilon_{1}(E)$ and $E^{2} \varepsilon_{2}(E)$ are presented in the form of interpolated functions $f_{1}$ and $f_{2}$ in formula (4), respectively. Such spectra were determined by variable angle spectroscopic ellipsometer (VASE) of J.A. Woollam Co. Inc. The example of such spectrum before differentiation is given in Fig. 1. The above mentioned algorithm was implemented in Program Mathematica 5.2 of Wolfram Company. By changing the $q$ value (fractional order of derivative) in Eq. (4) we are looking for the corresponding FDS. The example of such fractional spectra $\left(\mathrm{d}^{q} E^{2} \varepsilon(E) / \mathrm{d}^{q} E\right)$ for $q=0.75$ and $q=0.77$ are presented in Figs. 2 and 3. On the basis of FDS we can precisely extract the critical parameters $(\mathrm{CP})$ for the investigated sample.

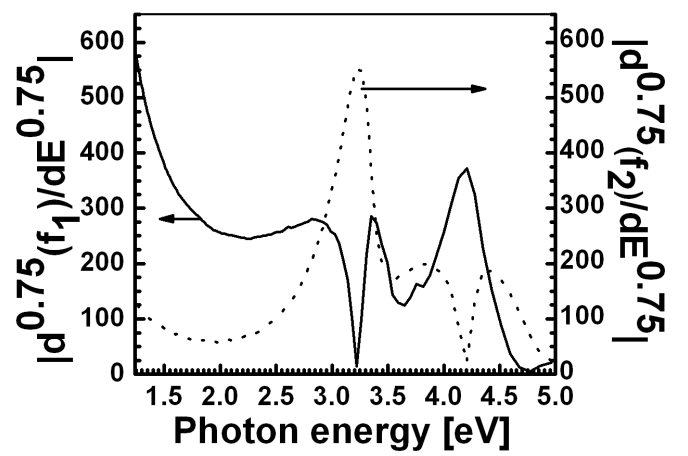

Fig. 2. FDS for $q=0.75$ for nitrogen implanted silicon substrates.

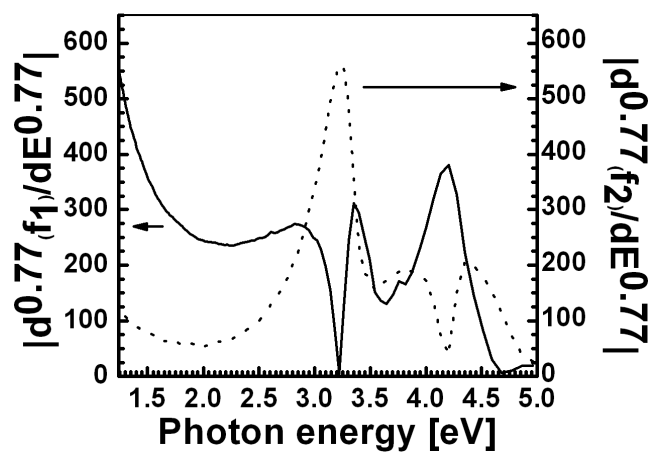

Fig. 3. FDS for $q=0.77$ for nitrogen implanted silicon substrates.

On the basis of the spectrum illustrated in Fig. 2 the extracted value of critical point $E_{1}$ was equaled to ca. $3.22 \mathrm{eV}$. Two extrema (minimum of FDS for $f_{1}$ function and maximum of FDS for $f_{2}$ function) of FDSsoccur at the same value of photon energy. In the vicinity of $E_{1}$ critical point, two peaks of FDS for $f_{1}$ function (two maxima) should be symmetrical and have the amplitude equaled to a half of the value of the amplitude of FDS for $f_{2}$ function.

On the basis of the spectrum illustrated in Fig. 3 the extracted value of critical point $E_{2}$ was equaled to ca. $4.20 \mathrm{eV}$. Two extrema (maximum of FDS for $f_{1}$ function and minimum of FDS for $f_{2}$ function) of FDSs occur at the same value of photon energy. In the vicinity of $E_{2}$ critical point, two peaks of FDS for $f_{2}$ function (two maxima) should be symmetrical and have the amplitude equaled to a half of the value of the amplitude of FDS for $f_{1}$ function.

\section{Optical spectra analyses of treated substrates}

For optical analysis of the implanted (111) oriented silicon substrates of $n$ conduction type by nitrogen were used. Procedures of preparation of these samples were described in the paper [8]. Also, the samples subjected to annealing at $1130{ }^{\circ} \mathrm{C}$ under high pressure at $1.1 \mathrm{GPa}$ in argon atmosphere for up to $5 \mathrm{~h}$ (high temperature- 
high pressure, HT-HP treatment [8]) after implantation process were investigated.

It is well known that sputter etching in $\mathrm{Ar}^{+}$plasma is an effective and promising method of in situ surface preparation prior to metal deposition if ion-induced damage of semiconductor surface could be minimized [9]. However, low energy $\mathrm{Ar}^{+}$ion etching can modify not only the surface morphology but also the surface composition [10]. In these experiments the optical spectra of (100) GaAs wafers prepared according to procedure described in [11] were analyzed.

\subsection{Si substrate}

Two characteristic critical points in silicon were investigated in this paper: point $E_{1}-E_{0}^{\prime}$ and $E_{2}$. Two kinds of transitions in Si band structure between 3.0 and $3.5 \mathrm{eV}$ in the spectrum are observed. The first kind concerns $E_{1}$ transition occurring along directions $\Lambda$ in the Brillouin zone. The second kind concerns $E_{0}^{\prime}$ structure and stands for the most lower direct band gap located in $\Gamma$ point.

The higher energetic point between 4.0 and $4.5 \mathrm{eV}$ in the spectrum denoted as $E_{2}$ is ascribed to the area conforming or being in the vicinity of $X$ and $\Sigma$ points in the space of wave vector $k$. Such behavior indicates small "abnormality" in the right "shoulder" symmetric structure of $E_{2}$ caused probably by superpositions of neighboring critical points at different dimensionality, amplitude and broadening. However, it can be approximately treated as a one structure. The good visualization of $\mathrm{Si}$ band structure in the Brillouin zone can be found in [12].

The results of the optical analysis by FDS and spectroscopic ellipsometry are shown in Table I.

TABLE I

Extracted values of Van Hove singularities for treated and untreated silicon substrates.

\begin{tabular}{c|c|c|c|c|c}
\hline \hline Samples/data & Time of HT-HP & $E_{1}$ & Fractional derivative order & $E_{2}$ & Fractional derivative order \\
\hline Herzingeret al. [13] & $0 \mathrm{~h}$ & 3.3500 & 0.790 & 4.2700 & 0.900 \\
impl. Si & $0 \mathrm{~h}$ & 3.2208 & 0.750 & 4.2034 & 0.770 \\
$1000^{\circ} \mathrm{C}, 1.1 \mathrm{GPa}$ & $1 \mathrm{~h}$ & 3.3067 & 0.998 & 4.1333 & 0.935 \\
$1000^{\circ} \mathrm{C}, 1.1 \mathrm{GPa}$ & $5 \mathrm{~h}$ & 3.3067 & 1.025 & 4.2759 & 1.500
\end{tabular}

TABLE II

(100) GaAs surface state after different treatments.

\begin{tabular}{|c|c|c|c|c|c|}
\hline \multicolumn{2}{|c|}{ Surface treatment } & \multirow{2}{*}{\multicolumn{4}{|c|}{ Spectroscopic ellipsometry (for $\lambda=630 \mathrm{~nm}$ ) }} \\
\hline \multicolumn{2}{|c|}{ Ion etching } & & & & \\
\hline Power $[\mathrm{W}]$ & Time $[\mathrm{s}]$ & Model & $\begin{array}{c}\text { Thickness of superficial } \\
\text { layer }[\mathrm{nm}]\end{array}$ & Refractive index $n$ & $\begin{array}{l}\text { Extinction } \\
\text { coefficient } k\end{array}$ \\
\hline 250 & 30 & $\mathrm{~A}^{*}$ & $d_{1}=50.00$ & 3.901 & 0.400 \\
\hline 150 & 40 & $\mathrm{~A}$ & $d_{1}=40.00$ & 3.910 & 0.429 \\
\hline 70 & 20 & A & $d_{1}=10.90$ & 3.828 & 0.458 \\
\hline 70 & 30 & $\mathrm{~A}$ & $d_{1}=13.52$ & 3.843 & 0.456 \\
\hline 70 & 40 & $\mathrm{~A}$ & $d_{1}=13.90$ & 3.843 & 0.456 \\
\hline 70 & 90 & $\mathrm{~A}$ & $d_{1}=20.60$ & 3.854 & 0.450 \\
\hline \multirow{2}{*}{\multicolumn{2}{|c|}{$\begin{array}{l}\text { Wet etching in } 5 \% \mathrm{HCl} ; t=3 \mathrm{~min} \\
\bullet \text { Wet etching in } 5 \% \mathrm{HCl} ; \mathrm{t}=3 \mathrm{~min} \\
\bullet \bullet \mathrm{Ar}+\text { ion etching; } P=70 \mathrm{~W}, t=30 \mathrm{~s}\end{array}$}} & $\mathrm{~B}^{* *}$ & $d_{2}=2.02$ & 1.820 & 0 \\
\hline & & A & $d_{1}=10.00$ & 3.702 & 0.398 \\
\hline \multirow{2}{*}{\multicolumn{2}{|c|}{ Oxide on GaAs }} & B & $d_{2}=2.15$ & 1.802 & 0 \\
\hline & & & - & 3.839 & 0.208 \\
\hline
\end{tabular}

${ }^{*}$ model A - modified layer on GaAs substrate, ${ }^{* *}$ model B - oxide layer on GaAs substrate.

Significant differences can be observed between the silicon samples treated and untreated (the Herzinger data). The annealing at HT-HP as well as during $5 \mathrm{~h}$ causes the return to the values of critical points before treatment (see Table I, $E_{2} \mathrm{CP}$ ). It can be evidenced for recrystallization of implanted samples due to HT-HP process. 
Similar behavior of these samples was observed by the other optical techniques [8].

\subsection{GaAs substrate}

Two characteristic critical points in gallium arsenide were investigated in this paper: point $E_{1}-E_{1}+\Delta_{1}$ and $E_{0}^{\prime}-E_{2}$.

Two kinds of transitions in GaAs band structure between 2.8 and $3.2 \mathrm{eV}$ in the spectrum are observed. The first kind concerns $E_{1}$ transition. The second kind concerns $E_{1}+\Delta_{1}$ structure.

The higher energetic points between 4.3 and $5.0 \mathrm{eV}$ in the spectrum were denoted as $E_{0}^{\prime}$ and $E_{2}$. The good visualization of GaAs band structure can be found in $[14,15]$.

The results of ellipsometric investigations GaAs treated substrates are shown in Table II. But the results of optical analyses for untreated samples by FDS was illustrated in Figs. 4 and 5.

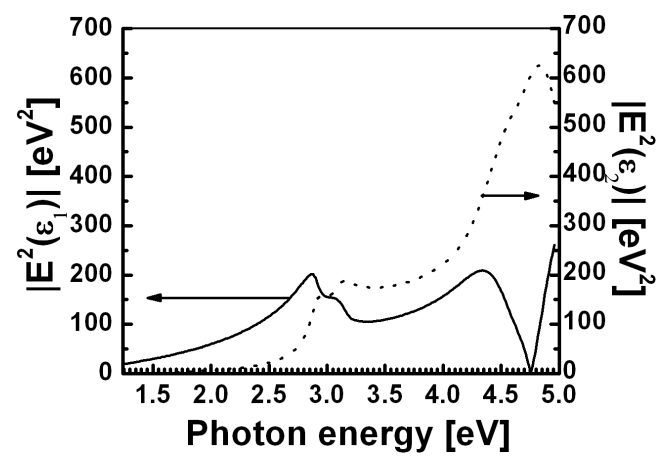

Fig. 4. Spectra $\left|\varepsilon_{1} E^{2}\right|$ and $\left|\varepsilon_{2} E^{2}\right|$ as a function of photon energy for gallium arsenide substrates.

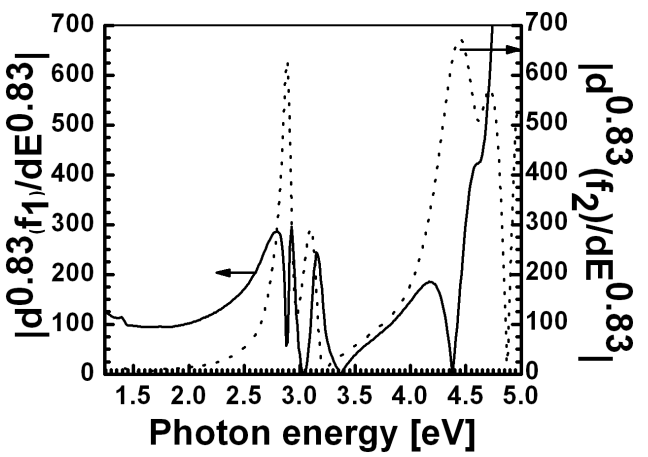

Fig. 5. FDS for $q=0.83$ for gallium arsenide substrates.
The ellipsometric results for untreated GaAs were similar to these obtained by Zollner [16].

\section{Conclusions}

Our studies portray that spectroscopic ellipsometry in combination with FDS stands for a very good tool to extract basic information on relevant physical quantities from the observed optical spectra, and it has the advantages of flexibility, directness, and sensitivity, which enable us to obtain the CP parameters efficiently with one consent. It concerns both semiconductors with direct (GaAs) and indirect band gap (Si).

\section{References}

[1] M.A. El-Sherbiny, H.H. El-Bahnasawy, M.M. El-Ocker, Nucl. Instrum. Methods. Phys. Res. B 168, $510(2000)$.

[2] K. Tao, T. Lai, Y. Zhang, Z. Yu, D. Mo, J. Phys., Condens. Matter 16, 3041 (2004).

[3] X.F. He, D. Mo, Chin. Phys. Lett. 3, 565 (1986).

[4] X.F. He, Phys. Rev. B 42, 11751 (1990).

[5] X.F. He, Phys. Rev. B 43, 2063 (1991).

[6] L.F. Lastras-Martinez, T. Ruf, M. Konuma, M. Cardona, Phys. Rev. B 61, 12946 (2000).

[7] K.B. Oldham, J. Spanier, The Fractional Calculus and Its Applications, Springer, Berlin 1976.

[8] W. Rzodkiewicz, A. Kudla, A. Misiuk, B. Surma, J. Bak-Misiuk, J. Hartwig, J. Ratajczak, Mater Sci. Semiconductor Proc. 7, 399 (2004).

[9] E. Papis-Polakowska, Electron Technology Int. J. 37/38, $1(2005 / 2006)$.

[10] R.V. Ghita, C. Negrilla, J. Opt. Adv. Mat. 5, 859 (2003).

[11] E. Papis, A. Baranska, P. Karbownik, A. Szerling, A. Wojcik-Jedlinska, M. Bugajski, W. Rzodkiewicz, J. Szade, A. Wawro, Opt. Appl., in press.

[12] J.R. Chelikovsky, M.L. Cohen, Phys. Rev. B 10, 5095 (1974).

[13] C.M. Herzinger, B. Johs, W.A. McGahan, J.A. Woollam, W. Paulson, J. Appl. Phys. 83, 3323 (1998).

[14] M. Rohlfing, P. Kruger, J. Pollmann, Phys. Rev. B 48, 17791 (1993).

[15] N.E. Christensen, Phys. Rev. B 30, 5753 (1984).

[16] S. Zollner, J. Appl. Phys. 90, 515 (2001). 